\title{
SPECT/CT: Standing on the Shoulders of Giants, It Is Time to Reach for the Sky!
}

\author{
Tim Van den Wyngaert ${ }^{1,2}$, Filipe Elvas ${ }^{1,2}$, Stijn De Schepper ${ }^{1,2}$, John A. Kennedy ${ }^{3,4}$, and Ora Israel ${ }^{4}$ \\ ${ }^{1}$ Nuclear Medicine, Antwerp University Hospital, Edegem, Belgium; ${ }^{2}$ Integrated Personalized and Precision Oncology Network, \\ Faculty of Medicine and Health Sciences, University of Antwerp, Wilrijk, Belgium; ${ }^{3}$ Department of Nuclear Medicine, Rambam \\ Healthcare Campus, Haifa, Israel; and ${ }^{4}$ Rappaport School of Medicine, Israel Institute of Technology, Haifa, Israel
}

Twenty years ago, SPECT/CT became commercially available, combining the strengths of both techniques: the diagnostic sensitivity of SPECT and the anatomic detail of CT. Other benefits initially included attenuation correction of SPECT reconstructions, ultimately evolving to correction techniques that would enable absolute tracer uptake quantification. Recent developments in SPECT hardware include solid-state digital systems with higher sensitivity and resolution, using novel collimator designs based on tungsten. Similar advances in CT technology have been introduced in hybrid SPECT/ CT systems, replacing low-end $x$-ray tubes with high-end multislice CT scanners equipped with iterative reconstruction, metal artifact reduction algorithms, and dual-energy capabilities. More recently, the design of whole-body SPECT/CT systems has taken another major leap with the introduction of a ring-shaped gantry equipped with multiple movable detectors surrounding the patient. These exciting developments have fueled efforts to develop novel SPECT radiopharmaceuticals, creating new chelators and prosthetic groups for radiolabeling. Innovative SPECT radionuclide pairs have now become available for radiolabeling with the potential for use as theranostic agents. The growth of precision medicine and the associated need for accurate radionuclide treatment dosimetry will likely drive the use of SPECT/CT in the near future. In addition, expanding clinical applications of SPECT/CT in other areas such as orthopedics offer exciting opportunities. Although it is true that the SPECT/CT ecosystem has seen several challenges during its development over the past 2 decades, it is now a feature-rich and mature tool ready for clinical prime time.

Key Words: radionuclide therapy; radiopharmaceuticals; SPECT/CT; development history; physics/engineering; tracer

J Nucl Med 2020; 61:1284-1291

DOI: 10.2967/jnumed.119.236943

$\mathbf{M}$ ark Twain is famously supposed to have said that history does not repeat itself, but it does often rhyme. With some adaptation, this can be said of the introduction of hybrid imaging modalities in nuclear medicine, in particular when comparing SPECT/CT with PET/CT. For both, their development was based on the premise that adding CT capabilities to the $\gamma$ - or PET

Received Mar. 2, 2020; revision accepted May 28, 2020.

For correspondence or reprints contact: Tim Van den Wyngaert, Department of Nuclear Medicine, Antwerp University Hospital, Wilrijkstraat 10, 2650 Edegem, Belgium.

E-mail: tim.van.den.wyngaert@uza.be

Published online Jul. 3, 2020.

COPYRIGHT @ 2020 by the Society of Nuclear Medicine and Molecular Imaging. camera would not only solve the problem of correcting for signal attenuation but also open the possibility of a 1-stop shop for functional and anatomic imaging.

Under the hybrid paradigm, the combined result exceeds the sum of its parts by the synergy in individual strengths of the techniques (the diagnostic sensitivity for SPECT and anatomic detail for $\mathrm{CT}$ ) while overcoming their mutually exclusive limitations (the diagnostic specificity for SPECT and sensitivity for CT). Despite these similarities, the speed at which both hybrid techniques were embraced in clinical practice and then generated literature evidence and impacted patient care has varied considerably. The significant differences in acceptance and implementation of the 2 hybrid modalities have sparked lively and contentious debates, assuming a winner-takes-all scenario (1).

Although PET/CT has been victorious in the sprint to market success, we will argue that in the marathon of progress in hybrid imaging, SPECT/CT has become an effective, versatile, and mature modality 20 years after its commercial introduction $(2,3)$. Its contribution to clinical progress in a wide spectrum of specialties was recently extensively reviewed (4). Instead, this paper will cover the SPECT/CT ecosystem by analyzing the lessons learned during its development and reviewing recent emerging applications, progress in physics/engineering and radiopharmacy/chemistry that will collectively drive its future (Fig. 1).

\section{PIONEERING EARLY YEARS}

The origins of SPECT and CT and the ultimate construction of the first integrated SPECT/CT devices have been reviewed in detail elsewhere, recognizing the tremendous academic efforts lying at its foundation (5). In particular, the pivotal work by Bruce H. Hasegawa's team at the University of California San Francisco merits special attention (6). The first commercially available SPECT/CT devices used low-performance X-ray tubes mounted on the same gantry as the SPECT detectors. This resulted in slow CT image acquisitions (up to $10 \mathrm{~min}$ ), limiting throughput and increasing the risk of motion artifacts, with suboptimal anatomic detail (7). Nonetheless, the improvements in SPECT quantification by virtue of attenuation correction and improved localization of areas of increased tracer uptake generated great enthusiasm. In particular, cardiac and oncologic imaging studies benefitted from the more reliable quantification and localization, respectively, as seen in the early published literature (Fig. 2A).

Subsequently, attenuation correction was recommended for routine clinical use, followed by broader professional procedural guidance for SPECT/CT (8). It also quickly became clear that major differences existed in the implementation of these techniques between 


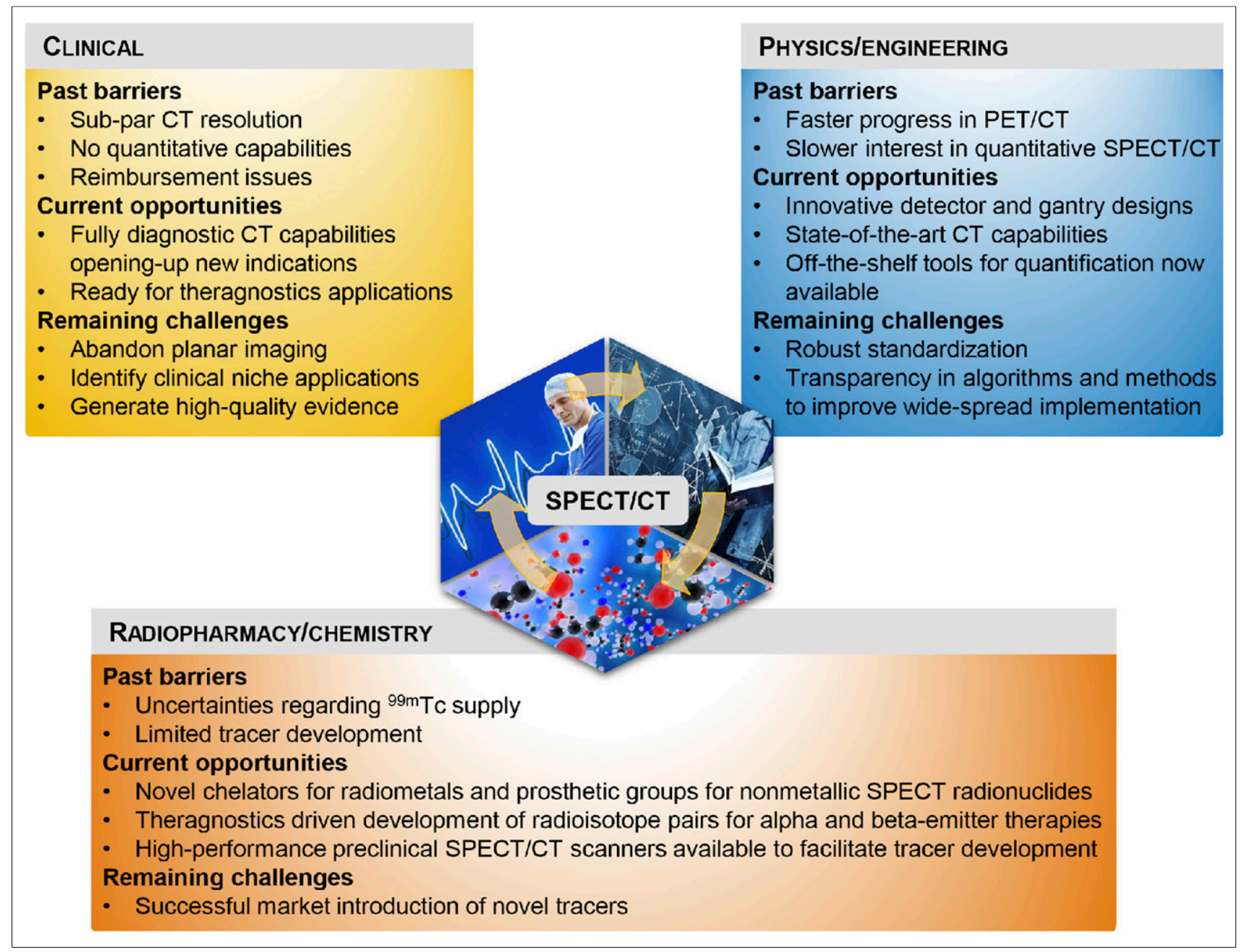

FIGURE 1. Summary of past barriers, current opportunities, and remaining challenges in developing SPECT/CT ecosystem, covering clinical, physics/engineering, and radiopharmacy/chemistry fields.

vendors and systems, leading to conflicting results in clinical use (9). At that time, the market had yet to decide which SPECT/CT design would ultimately prevail. This uncertainty fueled skepticism against

\section{NOTEWORTHY}

- Current state-of-the-art hybrid SPECT/CT devices offer significant improvements in SPECT spatial resolution and sensitivity, accurate absolute tracer uptake quantification comparable to PET/CT, and many advanced CT features.

- Technical progress is driving renewed interest in SPECT radiopharmaceutical development, with promising recent advances in chemistry and labeling methods.

- More widespread use of radionuclide therapies and theranostics requires robust patient dosimetry and is one of the driving applications of SPECT/CT use.

- Rigorous standardization of SPECT/CT is the next priority to achieve reproducible, consistent, and accurate quantification.

- Further multicenter, large-scale, prospective studies are needed to provide evidence-based data on the clinical value of SPECT/CT. the use of insufficiently validated and poorly standardized methods and contributed in part to a slow adoption of SPECT/CT in clinical practice (10). This was compounded by the rapid take-off of PET/CT, which had the advantage of being intrinsically quantitative and, in addition, was less prone to variation between vendors - or so it was perceived. It was a wake-up call that every innovation needs careful validation and standardization before clinical implementation, a lesson that reverberates even today (11).

\section{IF A LITTLE IS GREAT, IS MORE BETTER?}

Despite the clear anticipated advantages of SPECT/CT imaging, its clinical implementation was hampered by difficulties in defining key clinical areas of benefit. In the beginning it had been anticipated that only a fraction of patients would require a SPECT/ CT acquisition, making the incorporation of high-end CT systems hard to justify from a cost-to-benefit perspective. As a result, striking the balance between image quality, price, and safeguarding of patient throughput-all critical issues from a marketing point of view-proved difficult. Also, high-end CT capabilities were considered unnecessary given the low SPECT resolution and concerns regarding the CT-related additional radiation burden (12). On top of this, the flexibility in terms of the mechanical 


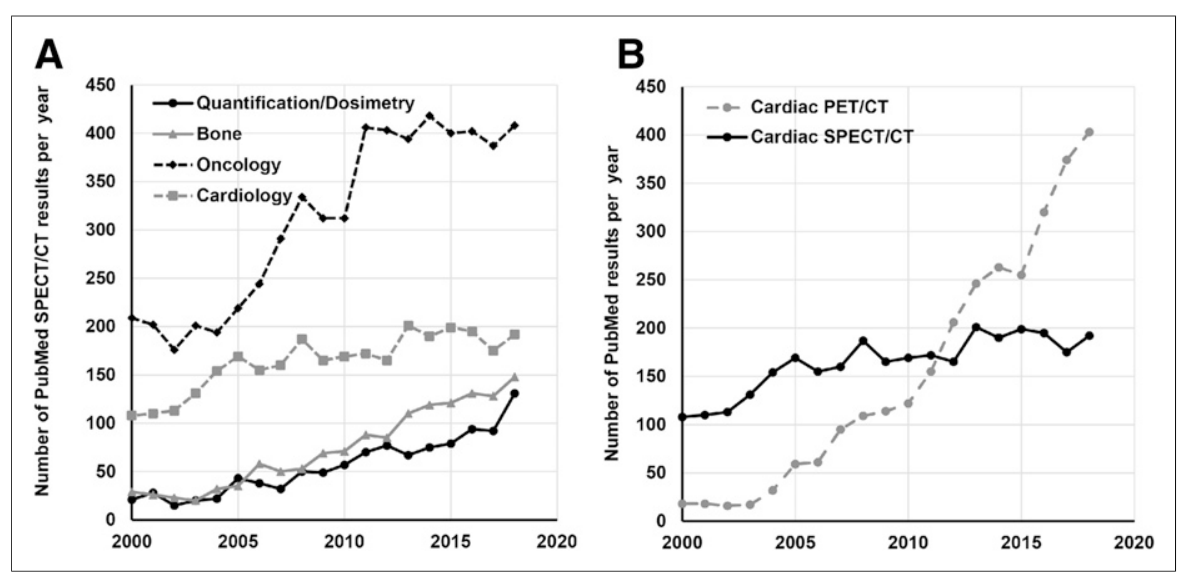

FIGURE 2. Total number of PubMed citations per year from 2000 to 2018 as measure of interest. (A) Publications using keyword SPECT/CT for selected subspecialty topics. (B) Publications using keyword SPECT/CT or PET/CT for cardiology.

design enabling detector movement, the supported target organs and regions, and the isotope energies demanded from SPECT systems remained higher than for PET/CT. In essence, SPECT/ CT devices had to offer familiarity and continuity to users in the clinical field on a level not demanded by early PET/CT systems. This required considerable trade-offs in gantry and collimator designs to maintain as much of this flexibility as possible, delaying major breakthroughs in improved SPECT resolution.

Even with its limited CT capabilities, the first-generation SPECT/ CT devices quickly produced exciting results in clinical practice (13). From 2004 onward, the introduction of multislice CT demonstrated the incremental benefits of better CT image quality (14). Sixty-fourslice CT capabilities emerged on high-end or dedicated SPECT/CT systems in 2006, mainly targeting cardiac applications (15). In practice, however, most users continued to use limited-capability CT until the end of the first decade of SPECT/CT. A lack of clinical imaging (Fig. 2B). guidelines defining what indications would benefit from hybrid SPECT/CT prolonged this situation.

This evolution was much easier for $\mathrm{PET} / \mathrm{CT}$, which at that time was-and arguably still remains-highly focused on whole-body acquisitions using a single-photon energy in a homogeneous, mostly oncology, clinical setting. In contrast to SPECT, attenuation correction is required for virtually all PET studies and CT enabled fast and robust correction methods. Indeed, PET/CT devices with 64-slice CT systems were commercially available from 2005 and quickly ended the era of PET-only cameras. These superior CT capabilities also impacted nononcologic indications and caused a shift in cardiac imaging from SPECT/ CT to PET/CT, an evolution that would prove difficult to reverse

The nuclear medicine community has struggled considerably in defining the appropriate type of CT required for SPECT/CT, leading to the appearance of confusing terminology such as lowdose or high-dose and nondiagnostic or diagnostic in CT acquisition protocols. Each of these terms is associated with perceived positive or negative connotations concerning radiation exposure and clinical value. This ambiguity has needlessly complicated defining the clinical role of SPECT/CT. With the benefit of hindsight, SPECT/CT could have profited from a different paradigm, where the appropriate CT acquisition parameters are tailored according to the specific disease or diagnostic question (Fig. 3) (16). Considerable effort will be required to harmonize clinical practice in this respect, with recent guidelines for bone SPECT/CT being a good initial attempt to define broad region-specific CT acquisition parameters (17).

\section{COMING OF AGE AND READY FOR PRIME TIME!}

\section{High-Level Features Coming Together}

From approximately 2010 onward, highend multislice CT variants have been part of all SPECT/CT portfolios. At the same time, iterative reconstruction techniques for CT were gradually introduced, reducing ionizing radiation doses up to $80 \%$ without loss of image quality and solving, in part, one of the initial barriers in the implementation of SPECT/CT (18). Around 2013, SPECT/CT as a hybrid modality had come of age, available with a full set of technical capabilities (enabling correction for attenuation, scatter, partial volume, and motion). This brought the potential for robust absolute quantification to clinical practice (Fig. 4), with reported accuracies for ${ }^{99 \mathrm{~m}} \mathrm{Tc}$ imaging to within $\pm 5 \%$ of the true radionuclide concentration $(19,20)$. Ironically, this technologic progress was briefly overshadowed by 


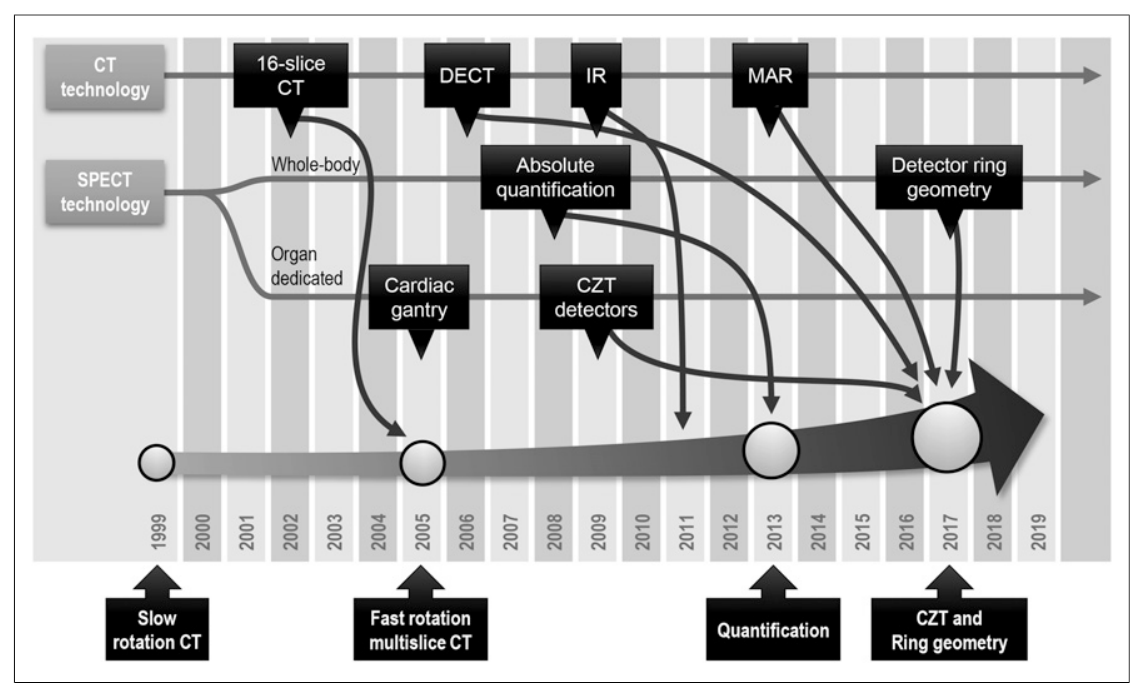

FIGURE 4. Summary of approximate time of introduction of milestones in whole-body SPECT/ CT technology, showing incorporation of subsequent innovations in CT and SPECT technology. Although some novel CT technologies were quickly incorporated into SPECT/CT systems (e.g., iterative reconstruction), others showed significant delays in their translation into hybrid scanners. For SPECT innovations, it is interesting to note parallel development of dedicated cardiac gantry designs that saw earlier introduction of breakthroughs, such as CZT detectors, compared with whole-body capable devices. DECT $=$ dual-energy $\mathrm{CT} ; \mathrm{IR}=$ iterative reconstruction; MAR $=$ metal artifact reduction.

a global ${ }^{99 \mathrm{~m}} \mathrm{Tc}$ shortage that created doubt on the viability of SPECT/ $\mathrm{CT}$ once more, as further discussed below.

\section{Precision Medicine as Breakthrough Application}

A renewed interest in radionuclide treatments using ${ }^{90} \mathrm{Y}$ and ${ }^{177} \mathrm{Lu}$ in oncology under the paradigm of precision medicine (popularized in nuclear medicine using the term theranostics) strongly relies on molecular tumor characterization and individualized treatment planning. Although ${ }^{90} \mathrm{Y}$ has been one of the main radionuclides used for therapy, it has recently been surpassed by ${ }^{177} \mathrm{Lu}$ in theranostics applications. The separate $\gamma$ - and $\beta$-decay modes of ${ }^{177} \mathrm{Lu}$ allow for imaging of the activity distribution and calculation of the dose distribution. It has now been recognized that individualized dosimetry is favored over fixed, or patient-weight- or body-surface-area-based, administered activities. Individualized dosimetry requires absolute activity measurements in order to deliver target doses that are as high as safely attainable and is possible only after correcting for degrading physical phenomena such as photon scatter, photon attenuation, partial-volume effect, and detector dead time (21). Increasingly accurate correction techniques and iterative reconstruction methods have improved the capabilities of modern SPECT/CT systems to achieve high-level quantitative images, comparable to PET/CT.

Tools to process an image in activity per unit volume have now become available, with acceptable accuracies in most organs and moderate-sized tumors. The in vivo accuracy of solid-state SPECT/ CT quantification of ${ }^{177} \mathrm{Lu}$-prostate-specific membrane antigen was recently reported to be within $20 \%$, with challenges remaining in quantifying smaller structures (22). It is expected that quantification and dosimetry applications will drive further applications of SPECT/ $\mathrm{CT}$, as seen in literature citations today (Fig. 2A).

\section{Leveraging the Full Diagnostic Power of SPECT/CT}

The improved capabilities of the embedded CT systems are benefitting the use of SPECT/CT in, for example, bone disease
(Fig. 2A). In addition, the novel multimodal SPECT reconstruction techniques can improve image resolution and have shown benefit in assessing uptake in small osseous structures (23). The availability of metal artifact reduction algorithms to reduce the degradation of CT images caused by photon-deprivation and beam-hardening artifacts has further improved the image quality of SPECT/CT. Another enhancement now available on SPECT/CT is dual-energy CT, which uses virtual monochromatic reconstructions for high-energy photons (140 $\mathrm{keV})$ that are less susceptible to metal artifacts (24). These breakthroughs have improved, among others, the assessment of painful arthroplasties, a diagnostic challenge increasing in prevalence due to changes in demographics. Moreover, SPECT/CT is less susceptible than MRI to image-degrading artifacts in patients after arthroplasty, even with the recent improvements in MRI metal artifact-reducing sequence technology.

The painful total-knee arthroplasty has been a successful model for developing the clinical application of high-end bone SPECT/CT. A consistent and relevant impact on patient management has been demonstrated, with the potential for health-care cost savings, making SPECT/CT part of the routine diagnostic algorithm for patients with pain after primary total-knee arthroplasty according to some leading groups $(25,26)$. Exciting results are emerging in other postoperative skeletal settings, including the spine, the hip, and hand or foot pain as well (27-29). Further progress will require novel radiopharmaceuticals offering improved pharmacokinetics (e.g., next-generation radiobisphosphonates) or visualizing specific causes such as infection (e.g., ${ }^{99 m}$ Tc-UBI-294), as further discussed below.

Despite the once gray-sky scenarios predicted by some for SPECT/CT imaging, current trends show a strong growth in the acceptance of state-of-the-art SPECT/CT devices. Recent data from Europe show a $22 \%$ increase in the number of installed SPECT/CT scanners in France from 2015 to 2018, with similar data from the United Kingdom and Germany available (30). Although SPECT/CT has come of age and is a reliable hybrid imaging modality ready for prime time, some unique challenges will need to be addressed. In particular, there are challenges regarding the use of SPECT/CT in young patients (e.g., for the assessment of low back pain) —an application that requires even more attention to exposure to ionizing radiation and regional issues with reimbursement pending further evidence of cost effectiveness.

\section{PROGRESS IN PHYSICS AND ENGINEERING}

\section{Innovation in Detector and Collimator Design}

Over the last few years, digital solid-state SPECT detector technology has become commercially available. Cadmium-zinc-telluride (CZT) detectors have excellent count sensitivity, system resolution, and energy resolution, enabling significant reductions in administered activities or acquisition time, as well as facilitating dynamic SPECT. Their superior energy resolution also facilitates dual-isotope imaging through improved separation of the photon peaks in the detected energy 
spectrum. The higher stopping power, on the other hand, allows for thinner detectors and a higher imaging resolution, on the order of $2.5 \mathrm{~mm}$ or even the submillimeter range for preclinical systems (31). Although these capabilities have been known for many years, issues such as slow and small crystal growth and crystalline defects hampered its cost-effective large-scale production-issues that have been solved only recently (32).

CZT itself is not a prerequisite of higher-resolution systems and has intrinsic detector efficiencies similar to traditional systems. Yet, the smaller footprint and lighter weight of these detectors facilitated the construction of gantry designs optimized for specific anatomic regions, focusing primarily on cardiac imaging. The improved spatial resolution and sensitivity with novel detector arrangements in CZT cardiac cameras (33) were facilitated by the introduction of more sophisticated reconstruction algorithms (e.g., Bayesian reconstruction) that could incorporate prior information about the expected characteristics of the image (34). For example, in one comparison between 2 dedicated cardiac CZT SPECT systems and the conventional dual-head Anger camera, both the central spatial resolution (6.7-8.6 vs. $15.0-15.3 \mathrm{~mm})$ and the count sensitivity on cardiac phantom images (460-850 vs. 130 counts $\mathrm{s}^{-1} \mathrm{MBq}^{-1}$ ) were superior with the CZT systems (33). At present, this technology is being incorporated into general-purpose dual- and multihead SPECT/CT system designs (Fig. 4) $(35,36)$. Most importantly, reducing image acquisition times and lowering the injected radionuclide activity are now possible for cardiac studies (37) and are being explored for a wider range of nuclear medicine studies.

The availability of compact detectors with higher intrinsic spatial resolution has enabled the implementation of novel collimator designs in the clinic $(37,38)$. This has been made possible by the use of tungsten and 3-dimensional printing techniques, delivering higher stopping power, smaller collimator bores, and reduced septal thickness. These new materials make possible new collimator geometries that, until now, had been beyond reach or impractical to implement on a large scale. Examples include minifying multiple-pinhole collimators (allowing more projections per detector) and (multiple-slit) slit-slat collimators (combining the advantages of pinhole and parallel-hole collimators). Both these approaches may be the basis for developing fully stationary detector systems, which could represent the next revolution in SPECT gantry design. The resulting clinical SPECT images achieve resolutions down to $3 \mathrm{~mm}$, with exciting preliminary clinical data in brain and cardiac imaging (39). However, the technique has not yet been implemented in hybrid SPECT/CT system designs.

\section{The Next Major Leap Forward}

A true paradigm shift in SPECT/CT design was unveiled in 2017, when the first $360^{\circ}$ ring-shaped gantry was introduced, equipped with 12 CZT-based elongated detectors that can move inward and outward to come as close to the patient as possible. These detectors are equipped with novel tungsten parallel-hole collimators and move in a swiveling motion to provide a unique scanning geometry (35). Although the clinical experience with this new device is still limited and the additional cost associated with this new design remains to be justified, preliminary results in bone imaging show that a whole-body SPECT acquisition of $20 \mathrm{~min}$ offers significant improvements in sharpness and contrast and can replace current bone-scanning protocols (40). This advance is also signaling an important and inevitable milestone in nuclear medicine practice: the end of the planar imaging era. Indeed, now is the time to let go of the paradigm of the hybrid $\gamma$-camera as the jack of all trades, master of none.

\section{Need for Standardization}

The recent technologic progress has created considerable variability among the available SPECT/CT systems, with respect to not only their physical designs but also the algorithms used for image correction and reconstruction. Such discrepancies may result in inconsistent or inaccurate results, limiting reproducibility, as seen early on with PET (41). Only rigorous standardization provided the solution to achieve reproducible, consistent, and accurate quantification. This required a common standard for quality control and quality assurance, as outlined in protocols by North American and European professional societies and groups $(42,43)$.

Multiple collaborative groups have started ambitious projects aimed at standardizing quantitative SPECT/CT and creating protocols to determine the accuracy and uncertainties of specific dosimetry platforms $(11,44)$. However, these have been hampered by a tendency to sacrifice user control of application settings and transparency in closed-source workstation solutions using proprietary algorithms in favor of easy-to-use interfaces. Other issues that complicate standardization are the increasing design differences between systems, the need to define the acceptable minimum level of image quality and, for the purpose of image quantification, the need to validate the use of (often small) phantoms as accurate surrogates of patient geometry.

These observations should prompt a call to action from major professional societies and international bodies such as the Society of Nuclear Medicine and Molecular Imaging, the European Association of Nuclear Medicine, and the International Atomic Energy Agency to launch initiatives supporting the standardization of SPECT/ CT quantification. Indeed, standardization and traceability are key requirements to harness the full potential of theranostic applications and provide truly individualized and high-quality patient care.

\section{INNOVATIONS AND CHALLENGES IN SPECT RADIOPHARMACEUTICAL DEVELOPMENT}

\section{Strength and Challenges of SPECT Radionuclides}

Improvements in SPECT/CT technology alone are not enough, as further adoption of the technique is critically linked to the development of novel SPECT radiopharmaceuticals. These remain highly attractive tools for imaging both in clinical practice and in preclinical research. A multitude of single-photon emitters is available (e.g., ${ }^{99 \mathrm{~m}} \mathrm{Tc},{ }^{111} \mathrm{In}$, and ${ }^{123} \mathrm{I}$ ) with half-lives longer than those of commonly used PET radionuclides, facilitating their distribution to more remote locations. Also, SPECT tracers are relatively inexpensive, often being available at a tenth of the cost of PET agents. Today, ${ }^{99 \mathrm{~m}} \mathrm{Tc}$ is used in approximately $85 \%$ of all nuclear medicine diagnostic procedures, accounting for 30 million examinations worldwide every year (45). However, its production depends on the use of highly enriched uranium targets in nuclear research reactors, making the global supply chain complicated and particularly vulnerable to reactor shutdowns. First in 2009, and then in 2012 and 2013, planned and unplanned shutdowns have caused extended global shortages. To safeguard future supply, providers have been encouraged to recover their full costs in order to support the transition to low-enriched-uranium targets and investments in sufficient production and reserve capacity in case of unplanned outages. At the same time, alternative or supplementary production technologies are also being developed, including 
neutron activation of ${ }^{98} \mathrm{Mo}$ targets in research reactors, ${ }^{99} \mathrm{Mo}$ production with linear accelerators, and direct production of ${ }^{99 \mathrm{~m}} \mathrm{Tc}$ on cyclotrons (46).

Beyond ${ }^{99 \mathrm{~m}} \mathrm{Tc}$, SPECT radionuclides with an extended half-life (i.e., ${ }^{111} \mathrm{In}$ and ${ }^{123} \mathrm{I}$ ) enable the use of lengthier and more complex radiolabeling procedures, as well as longer imaging protocols. A typical example is infection imaging using ${ }^{111}$ In-labeled white blood cells, a modality that benefits from delayed acquisitions to improve specificity (47). A unique strength of SPECT is dual-isotope imaging, which enables simultaneous detection of tracer distribution in space and over time in a single imaging session, thus reducing acquisition times (and therefore patient discomfort), image artifacts, and quantitation errors due to patient motion. This approach is particularly well suited for the study of interconnected functional, metabolic, chemical, and biologic processes. This methodology was recently used to study Takotsubo cardiomyopathy, showing that dual-isotope SPECT with the perfusion tracer $99 \mathrm{~m}$ Tc-MIBI and the fatty-acid metabolism imaging agent ${ }^{123}$ I-BMIPP ( ${ }^{123}$ I-betamethyl-piodophenyl-pentadecanoic acid) combined with cardiac CT could visualize the typical changes in both processes caused by vasospasms in the distal left anterior descending coronary artery (48).

\section{Innovation in SPECT Tracers for Imaging and Treatment}

Therapeutic drug development is targeting ever more specific pathways or processes using small-molecule inhibitors or monoclonal antibodies. Ideally, tracer development can mirror these versatile approaches to derive predictive diagnostic radiopharmaceuticals or to exploit the highly specific targeting properties of these compounds to produce radionuclide therapies.

The small-molecule prostate-specific membrane antigen inhibitor radiotracers have become an important class of compounds in the management of prostate cancer. One of the first compounds of this class to be developed was the radioiodinated $\left({ }^{123} \mathrm{I}\right)$ tracer MIP-1095, which has successfully been used for targeted tumor therapy (49). Uniquely, MIP-1095 can be used as a theranostic agent for SPECT $\left({ }^{123} \mathrm{I}\right)$ and radionuclide therapy $\left({ }^{131} \mathrm{I}\right)$. In addition, the ${ }^{99 \mathrm{~m}} \mathrm{Tc}$-analog MIP-1404 (also known as ${ }^{99 \mathrm{~m}}$ Tc-trofolastat), prepared using the technetium tricarbonyl core, has been proposed for SPECT/CT imaging, has shown good tumor targeting ability and favorable biodistribution, and has recently entered a phase 3 study to support possible market introduction (50).

For the development of monoclonal antibody or peptide-based imaging agents, nonmetallic radionuclides such as ${ }^{123} \mathrm{I}$ have frequently been labeled using direct electrophilic aromatic substitution on histidine or tyrosine amino acid residues. Unfortunately, the required labeling conditions are often too harsh for antibodies and may damage their structure and affinity. This problem has recently been overcome by the introduction of reactive prosthetic groups, such as the novel radioiodinated BODIPY dual functional agent, allowing labeling under milder conditions. Proof-of-concept data demonstrated successful SPECT imaging with ${ }^{123} \mathrm{I}$-trastuzumab of HER2-positive tumors (51).

For metallic SPECT radionuclides such as ${ }^{99 \mathrm{~m} T c}$ and ${ }^{111} \mathrm{In}$, bifunctional chelating groups have been the cornerstone for labeling antibodies and peptides. The versatile chemistry of ${ }^{99 \mathrm{~m}} \mathrm{Tc}$, with its stable and readily accessible oxidation states, is characterized by chemically robust core structures that can be exploited as platforms for radiopharmaceutical design. One such well-known approach uses the ${ }^{99 \mathrm{~m}} \mathrm{Tc}(\mathrm{V})$-organohydrazino (HYNIC) core to derivatize a wide range of different targeting molecules (52). A promising example is the development of ${ }^{99 \mathrm{~m}} \mathrm{Tc}$-duramycin as a
SPECT tracer for apoptosis, enabling imaging of the induction of cell death early after chemotherapy and radiotherapy. Providing a very early readout of treatment effect, it could potentially be used to tailor cancer treatments more quickly, sparing toxicity and cost in nonresponding patients (53). Another interesting target in oncology and cardiovascular medicine is angiogenesis, with ${ }^{99 \mathrm{~m}} \mathrm{Tc}-$ HYNIC-L(arginine-glycine-aspartate) or ${ }^{99 \mathrm{~m} T c-m a r a c i c l a t i d e ~ i n t e g r i n ~}$ $\alpha_{v} \beta_{3}$ imaging demonstrating the ability to visualize tumors and treatment response in cancer models (54). Integrin imaging has also been used to visualize inflamed atherosclerotic plaques, with the potential to noninvasively identify high-risk plaques in patients (55). Finally, expression of the c-Met receptor in non-small cell lung cancer could recently be demonstrated with ${ }^{99 \mathrm{~m}} \mathrm{Tc}-\mathrm{HYNIC}-\mathrm{c}-\mathrm{Met}-$ binding peptide SPECT, providing essential information on a signaling pathway that contributes to cancer progression and may mediate acquired resistance to epidermal growth factor receptor-targeted therapy (56). A multitude of SPECT/CT applications and their potential advantages can be envisioned, targeting a wide range of important predictive tumor patterns aiding treatment selection and available in convenient kit formulations.

A drawback of the HYNIC platform is that its chemistry is often complex, hampering clinical translation because of regulatory issues. A possible solution is the introduction of the organometallic $\left[{ }^{99 \mathrm{~m}} \mathrm{Tc}(\mathrm{CO})_{3}\right]^{+}$core. It is a versatile building block, and the immediate water-soluble precursor $\left[{ }^{99 \mathrm{~m}} \mathrm{Tc}(\mathrm{CO})_{3}\left(\mathrm{H}_{2} \mathrm{O}\right)_{3}\right]^{+}$can easily be obtained from homemade or commercially available kits. A major advantage of labeling with the ${ }^{99 \mathrm{~m}} \mathrm{Tc}$-tricarbonyl core is the very wide variety of ligands that bind efficiently to $\mathrm{Tc}(\mathrm{I})$, forming highly robust complexes. Recently, fibroblast-activating protein inhibitors were successfully labeled using this method, enabling SPECT/CT imaging of cancer-associated fibroblasts, present in many malignancies. Because fibroblast-activating protein is typically not expressed in healthy tissues, it may represent an important target for theranostic applications (57).

Single-domain antibodies have also proven to be particularly suited for ${ }^{99 \mathrm{~m}} \mathrm{Tc}$ labeling via tricarbonyl chemistry, with many exciting possible applications, including the noninvasive quantification of immune checkpoint (programmed death-ligand 1) expression in tumors to guide immunotherapy (58).

Progress is also being made in improving surrogate radionuclides used for pretherapy SPECT/CT imaging and dosimetry studies. Although ${ }^{111} \mathrm{In}$ is still frequently used for this purpose, its chemistry is slightly different from that of the radiolanthanides typically coordinated in these ligands (e.g., ${ }^{177} \mathrm{Lu}$ ). This difference may introduce errors in assessing the tissue distribution and could be solved using the radiolanthanide ${ }^{155} \mathrm{~Tb}(59)$. The administration of ${ }^{155} \mathrm{~Tb}$ alongside a therapeutic terbium isotope, such as ${ }^{149} \mathrm{~Tb}$ or ${ }^{161} \mathrm{~Tb}$, would represent the ideal theranostic pair because of the identical chemical properties. Similarly, the cyclotron-produced SPECT radioisotope ${ }^{203} \mathrm{~Pb}$ is attracting attention as the imaging surrogate for the therapeutic ${ }^{212} \mathrm{~Pb}$, as both can be directly imaged using SPECT/CT (60). These new diagnostic-therapeutic pairs may greatly facilitate the calculation of the dosimetry of $\alpha$ - and $\beta$-emitter-labeled radiopharmaceuticals. It is clear that recent innovations in radiopharmaceutical and chemistry techniques have created a promising and versatile landscape of powerful labeling methods that show great opportunity in developing the next generation of SPECT/CT radiopharmaceuticals. 


\section{CONCLUSION}

The last 20 years have witnessed a paradigm shift with the introduction of SPECT/CT as a hybrid imaging modality in nuclear medicine. The development of SPECT/CT has often been benchmarked against that of PET/CT. However, the appropriate use of SPECT/CT needs to consider a variety of options in the clinical setting, including the availability of various radionuclides, the need to image one or more body regions using optimized acquisition modes, and adherence to appropriate-use criteria for the multitude of oncologic and nononcologic indications. These inherent differences between the 2 modalities render such comparisons meaningless as a way to value the relative success of both techniques. The SPECT/CT ecosystem has seen its challenges but has emerged over the past 2 decades as a feature-rich and mature tool ready for clinical prime time, because of innovations in physics and engineering and in radiopharmacy and chemistry. Nevertheless, additional efforts should be made to standardize the quantitative results of contemporary SPECT/CT technology. Furthermore, a continued research effort is required to value its clinical benefit and assess its cost effectiveness. Proof of benefit in economic and humanistic outcomes is critical in order to gain widespread adoption of SPECT/CT.

\section{DISCLOSURE}

Ora Israel is a consultant for GE Healthcare. No other potential conflict of interest relevant to this article was reported.

\section{REFERENCES}

1. Alavi A, Basu S. Planar and SPECT imaging in the era of PET and PET-CT: can it survive the test of time? Eur J Nucl Med Mol Imaging. 2008;35:1554-1559.

2. Bocher M, Balan A, Krausz Y, et al. Gamma camera-mounted anatomical x-ray tomography: technology, system characteristics and first images. Eur J Nucl Med. 2000;27:619-627.

3. Patton JA, Delbeke D, Sandler MP. Image fusion using an integrated, dual-head coincidence camera with x-ray tube-based attenuation maps. J Nucl Med. 2000; 41:1364-1368.

4. Israel O, Pellet O, Biassoni L, et al. Two decades of SPECT/CT: the coming of age of a technology - an updated review of literature evidence. Eur J Nucl Med Mol Imaging. 2019;46:1990-2012.

5. Hutton BF. The origins of SPECT and SPECT/CT. Eur J Nucl Med Mol Imaging. 2014;41(suppl 1):S3-S16.

6. Lang TF, Hasegawa BH, Liew SC, et al. Description of a prototype emission-transmission computed tomography imaging system. J Nucl Med. 1992;33:1881-1887.

7. Even-Sapir E, Keidar Z, Sachs J, et al. The new technology of combined transmission and emission tomography in evaluation of endocrine neoplasms. $\mathrm{J} \mathrm{Nucl}$ Med. 2001;42:998-1004.

8. Hendel RC, Corbett JR, Cullom SJ, DePuey EG, Garcia EV, Bateman TM. The value and practice of attenuation correction for myocardial perfusion SPECT imaging: a joint position statement from the American Society of Nuclear Cardiology and the Society of Nuclear Medicine. J Nucl Cardiol. 2002;9: $135-143$.

9. O'Connor M, Kemp B, Anstett F, et al. A multicenter evaluation of commercial attenuation compensation techniques in cardiac SPECT using phantom models. J Nucl Cardiol. 2002;9:361-376.

10. Wackers FJ. Attenuation correction, or the emperor's new clothes? J Nucl Med. 1999;40:1310-1312.

11. Peters SMB, van der Werf NR, Segbers M, et al. Towards standardization of absolute SPECT/CT quantification: a multi-center and multi-vendor phantom study. EJNMMI Phys. 2019;6:29.

12. Bach-Gansmo T, Schwarzlmuller T, Joraholmen V, et al. SPECT/CT hybrid imaging; with which CT? Contrast Media Mol Imaging. 2010;5:208-212.

13. Keidar Z, Israel O, Krausz Y. SPECT/CT in tumor imaging: technical aspects and clinical applications. Semin Nucl Med. 2003;33:205-218.

14. Römer W, Nomayr A, Uder M, Bautz W, Kuwert T. SPECT-guided CT for evaluating foci of increased bone metabolism classified as indeterminate on SPECT in cancer patients. J Nucl Med. 2006;47:1102-1106.
15. Keidar Z, Bar-Shalom R, Rispler S, et al. Hybrid cardiac SPECT/64-slice CTCA imaging: technical feasibility and initial clinical experience [abstract]. $\mathrm{J} \mathrm{Nucl}$ Med. 2007;48(suppl):102P.

16. Rehani MM, Szczykutowicz TP, Zaidi H. CT is still not a low-dose imaging modality. Med Phys. 2020;47:293-296.

17. Van den Wyngaert T, Strobel K, Kampen WU, et al. The EANM practice guidelines for bone scintigraphy. Eur J Nucl Med Mol Imaging. 2016;43:1723-1738.

18. Sibille L, Chambert B, Alonso S, et al. Impact of the adaptive statistical iterative reconstruction technique on radiation dose and image quality in bone SPECT/ CT. J Nucl Med. 2016;57:1091-1095.

19. Bailey DL, Willowson KP. Quantitative SPECT/CT: SPECT joins PET as a quantitative imaging modality. Eur J Nucl Med Mol Imaging. 2014;41(suppl 1): S17-S25.

20. De Schepper S, Ritt P, Van den Wyngaert T, Kuwert T. Quantitative radionuclide imaging of bone metastases. Q J Nucl Med Mol Imaging. 2019;63:129-135.

21. Chiesa C, Sjogreen Gleisner K, Flux G, et al. The conflict between treatment optimization and registration of radiopharmaceuticals with fixed activity posology in oncological nuclear medicine therapy. Eur J Nucl Med Mol Imaging. 2017;44:1783-1786.

22. Kennedy JA, Lugassi R, Gill R, Keidar Z. Digital solid-state SPECT/CT quantitation of absolute ${ }^{177} \mathrm{Lu}$-radiotracer concentration: in vivo/in vitro validation. J Nucl Med. February 28, 2020 [Epub ahead of print].

23. Delcroix O, Robin P, Gouillou M, et al. The new SPECT/CT reconstruction algorithm xSPECT Bone ${ }^{\circledR}$ : reliability and accuracy in daily practice for nononcologic bone diseases [abstract]. J Nucl Med. 2017;58(suppl):1213.

24. McCollough CH, Leng S, Yu L, Fletcher JG. Dual- and multi-energy CT: principles, technical approaches, and clinical applications. Radiology. 2015;276:637-653.

25. Murer AM, Hirschmann MT, Amsler F, Rasch H, Huegli RW. Bone SPECT/CT has excellent sensitivity and specificity for diagnosis of loosening and patellofemoral problems after total knee arthroplasty. Knee Surg Sports Traumatol Arthrosc. 2020;28:1029-1035.

26. Van den Wyngaert T, Palli SR, Imhoff RJ, Hirschmann MT. Cost-effectiveness of bone SPECT/CT in painful total knee arthroplasty. J Nucl Med. 2018;59:17421750 .

27. Gnanasegaran G, Paycha F, Strobel K, et al. Bone SPECT/CT in postoperative spine. Semin Nucl Med. 2018;48:410-424.

28. Kampen WU, Westphal F, Van den Wyngaert T, et al. SPECT/CT in postoperative foot and ankle pain. Semin Nucl Med. 2018;48:454-468.

29. Van den Wyngaert T, Paycha F, Strobel K, et al. SPECT/CT in postoperative painful hip arthroplasty. Semin Nucl Med. 2018;48:425-438.

30. Les enquetes nationales de la SFMN: activites de la medecine nucleaire moyens humains \& materiels_annee 2018. Société Française de Médecine Nucléaire website. https://www.sfmn.org/drive/SECRETARIAT\%20GENERAL/ENQUETE_ ANNUELLE/EnqueteNationale2019_publicWeb.pdf. Accessed July 1, 2020.

31. Matsunari I, Miyazaki Y, Kobayashi M, et al. Performance evaluation of the eXplore speCZT preclinical imaging system. Ann Nucl Med. 2014;28:484-497.

32. Mackenzie J, Chen H, Awadalla SA, et al. Recent advances in THM CZT for nuclear radiation detection [abstract]. MRS Proceedings. 2009;1164:1164-L1004.

33. Imbert L, Poussier S, Franken PR, et al. Compared performance of high-sensitivity cameras dedicated to myocardial perfusion SPECT: a comprehensive analysis of phantom and human images. J Nucl Med. 2012;53:1897-1903.

34. Green PJ. Bayesian reconstructions from emission tomography data using a modified EM algorithm. IEEE Trans Med Imaging. 1990;9:84-93.

35. Desmonts C, Bouthiba MA, Enilorac B, Nganoa C, Agostini D, Aide N. Evaluation of a new multipurpose whole-body CzT-based camera: comparison with a dual-head Anger camera and first clinical images. EJNMMI Phys. 2020;7:18.

36. Yamane T, Kondo A, Takahashi M, et al. Ultrafast bone scintigraphy scan for detecting bone metastasis using a CZT whole-body gamma camera. Eur J Nucl Med Mol Imaging. 2019;46:1672-1677.

37. Herzog BA, Buechel RR, Katz R, et al. Nuclear myocardial perfusion imaging with a cadmium-zinc-telluride detector technique: optimized protocol for scan time reduction. $J$ Nucl Med. 2010;51:46-51.

38. Van Audenhaege K, Van Holen R, Vandenberghe S, Vanhove C, Metzler SD, Moore SC. Review of SPECT collimator selection, optimization, and fabrication for clinical and preclinical imaging. Med Phys. 2015;42:4796-4813.

39. Chen Y, Goorden MC, Vastenhouw B, Beekman FJ. Optimized sampling for high resolution multi-pinhole brain SPECT with stationary detectors. Phys Med Biol. 2020;65:015002.

40. Imbert L, Chevalier E, Claudin M, et al. A one-shot whole-body bone SPECT may be recorded in less than 20 minutes with the high-sensitivity Veriton ${ }^{\circledR}$ CZTcamera [abstract]. J Nucl Med. 2019;60(suppl):1288. 
41. Geworski L, Knoop BO, de Wit M, Ivancevic V, Bares R, Munz DL. Multicenter comparison of calibration and cross calibration of PET scanners. J Nucl Med. 2002;43:635-639.

42. Boellaard R, Willemsen AT, Arends B, Visser EP. EARL procedure for assessing PET/CT system specific patient FDG activity preparations for quantitative FDG PET/CT studies. Research 4 Life website. http://earl.eanm.org/html/img/pool/ EARL-procedure-for-optimizing-FDG-activity-for-quantitative-FDG-PET-studies_ version_1_1.pdf. Accessed July 1, 2020.

43. Sunderland JJ, Christian PE. Quantitative PET/CT scanner performance characterization based upon the Society of Nuclear Medicine and Molecular Imaging Clinical Trials Network oncology clinical simulator phantom. J Nucl Med. 2015;56: 145-152.

44. Gregory RA, Murray I, Gear J, et al. Standardised quantitative radioiodine SPECT/CT imaging for multicentre dosimetry trials in molecular radiotherapy. Phys Med Biol. 2019;64:245013.

45. Nuclear Energy Agency. The Supply of Medical Radioisotopes: Medical Isotope Demand and Capacity Projection for the 2018-2023 Period. Paris, France: OECD Nuclear Energy Agency; 2018.

46. Ruth TJ. The medical isotope crisis: how we got here and where we are going. J Nucl Med Technol. 2014;42:245-248.

47. Signore A, Jamar F, Israel O, Buscombe J, Martin-Comin J, Lazzeri E. Clinical indications, image acquisition and data interpretation for white blood cells and anti-granulocyte monoclonal antibody scintigraphy: an EANM procedural guideline. Eur J Nucl Med Mol Imaging. 2018;45:1816-1831.

48. Sugihara Y, Fukushima Y, Kumita SI, Takano H, Shimizu W. Diagnostic performance of hybrid cardiac SPECT/CT imaging for patients with Takotsubo cardiomyopathy. Eur J Hybrid Imaging. 2018;2:5.

49. Hillier SM, Maresca KP, Femia FJ, et al. Preclinical evaluation of novel glutamate-urea-lysine analogues that target prostate-specific membrane antigen as molecular imaging pharmaceuticals for prostate cancer. Cancer Res. 2009;69: 6932-6940.

50. Goffin KE, Joniau S, Tenke P, et al. Phase 2 study of ${ }^{99 \mathrm{~m}}$ Tc-trofolastat SPECT/ $\mathrm{CT}$ to identify and localize prostate cancer in intermediate- and high-risk patients undergoing radical prostatectomy and extended pelvic LN dissection. $J$ Nucl Med. 2017;58:1408-1413.

51. Ono M, Watanabe H, Ikehata Y, et al. Radioiodination of BODIPY and its application to a nuclear and optical dual functional labeling agent for proteins and peptides. Sci Rep. 2017;7:3337.

52. Liu S. The role of coordination chemistry in the development of target-specific radiopharmaceuticals. Chem Soc Rev. 2004;33:445-461.

53. Elvas F, Boddaert J, Vangestel C, et al. ${ }^{99 \mathrm{~m} T c-d u r a m y c i n ~ S P E C T ~ i m a g i n g ~ o f ~}$ early tumor response to targeted therapy: a comparison with ${ }^{18} \mathrm{~F}-\mathrm{FDG}$ PET. J Nucl Med. 2017;58:665-670.

54. Cook GJR, Azad GK, Taylor BP, et al. Imaging $\alpha v \beta 3$ integrin expression in skeletal metastases with ${ }^{99 \mathrm{~m}}$ Tc-maraciclatide single-photon emission computed tomography: detection and therapy response assessment. Eur J Nucl Med Mol Imaging. 2018;45:898-903.

55. Vancraeynest D, Roelants V, Bouzin C, et al. $\alpha v \beta 3$ integrin-targeted microSPECT/CT imaging of inflamed atherosclerotic plaques in mice. EJNMMI Res. 2016;6:29

56. Han Z, Xiao Y, Wang K, et al. Development of a SPECT tracer to image c-Met expression in a xenograft model of non-small cell lung cancer. J Nucl Med. 2018;59:1686-1691.

57. Lindner T, Altmann A, Kraemer S, et al. Design and development of ${ }^{99 \mathrm{~m} T \mathrm{~T}}$ labeled FAPI-tracers for SPECT-imaging and ${ }^{188}$ Re therapy. J Nucl Med. March 13, 2020 [Epub ahead of print].

58. Broos K, Keyaerts M, Lecocq Q, et al. Non-invasive assessment of murine PDL1 levels in syngeneic tumor models by nuclear imaging with nanobody tracers. Oncotarget. 2017;8:41932-41946.

59. Müller C, Fischer E, Behe M, et al. Future prospects for SPECT imaging using the radiolanthanide terbium-155: production and preclinical evaluation in tumorbearing mice. Nucl Med Biol. 2014;41(suppl):e58-e65.

60. Dos Santos JC, Schafer M, Bauder-Wust U, et al. Development and dosimetry of ${ }^{203} \mathrm{~Pb} /{ }^{212} \mathrm{~Pb}$-labelled PSMA ligands: bringing "the lead" into PSMA-targeted alpha therapy? Eur J Nucl Med Mol Imaging. 2019;46:1081-1091. 